\title{
Knockdown of GC binding factor 2 by RNA interference inhibits invasion and migration of vascular smooth muscle cells
}

\author{
YING MA $^{1,2^{*}}$, YONGQIANG REN ${ }^{2 *}$ and JUN GUAN ${ }^{2}$ \\ ${ }^{1}$ Qingdao University, Qingdao, Shandong 266073; ${ }^{2}$ Department of Cardiology, \\ Qingdao Municipal Hospital (Group), Qingdao, Shandong 266034, P.R. China
}

Received November 22, 2018; Accepted May 17, 2019

DOI: $10.3892 / \mathrm{mmr} .2019 .10410$

\begin{abstract}
GC binding factor 2 (GCF2) is a transcriptional repressor that inhibits the transcription of GC-rich promoters, thereby regulating biological processes, including proliferation. However, the role of GCF2 in vascular smooth muscle cells (VSMCs) remains unclear. The level of $\alpha$-smooth muscle $(\alpha-\mathrm{SM})$ actin was determined by immunofluorescence. Cell viability, migration and invasion were analyzed using Cell Counting Kit-8, wound healing and Transwell assays, respectively. Apoptosis and cell cycle progression were determined using flow cytometry. The expressions of Bcl-2, Bax, cleaved caspase-3, cyclin E, CDK2 and the CDK inhibitor p21 were determined by reverse transcription-quantitative (RT-q) PCR and western blot analysis. RT-qPCR was performed to analyze the levels of GCF2 and western blot analysis was conducted to determine the phosphorylation levels of PI3K and AKT. $\alpha-S M$ actin was found to be expressed in VSMCs. Cell viability, migration and invasion were inhibited by small interfering (si)RNA targeting GCF2. Changes in the expression levels of Bcl-2, Bax and cleaved caspase- 3 showed that the pro-apoptotic capacity of the cells was increased by siGCF2. Cell cycle arrest in the $G_{0} / G_{1}$ phase was induced by siGCF2, which was accompanied by changes in the levels of cyclin $\mathrm{E}$, CDK2 and p21. Furthermore, phosphorylation of PI3K and AKT was suppressed by siGCF2. However, the inhibitory effects of siGCF2 on cell viability, migration and invasion were increased by insulin-like growth factor 1 , which is a specific agonist of AKT. The anti-proliferative activity of siGCF2 may be associated with the PI3K/AKT pathway in VSMCs.
\end{abstract}

Correspondence to: Dr Jun Guan, Department of Cardiology, Qingdao Municipal Hospital (Group), 1 Jiaozhou Road, Qingdao, Shandong 266034, P.R. China

E-mail: guanjun_jguan@163.com

${ }^{*}$ Contributed equally

Key words: GC-binding factor 2, vascular smooth muscle cell, invasion, migration

\section{Introduction}

Abnormal invasion and migration of vascular smooth muscle cells (VSMCs) leads to the thickening of the arterial intima, a common pathological basis for the development of cardiovascular disease (1). VSMCs have substantial plasticity, allowing the cells to quickly adapt to changes in the surrounding environment (2). Changes in vascular smooth muscle phenotype play an important role in cardiovascular disease, including atherosclerosis and restenosis after percutaneous coronary angioplasty (3). Previous studies have suggested that VSMCs have both systolic and synthetic phenotypes, and that the normal arterial wall is mainly composed of contractile VSMCs, which are present in adult blood vessels and are accompanied by high protein expression (for example, smooth muscle tendon-actin was found to be highly expressed). This previous research also found that VSMCs play an important role in maintaining vascular tone and homeostasis, and have a low potential for proliferation and migration $(4,5)$. By contrast, in pathological states, the VSMCs found in the intima are mainly synthetic, exhibiting reduced expression of contraction-related proteins. However, VSMCs have great potential for proliferation and migration in pathological states (6). Therefore, exploring the molecular mechanisms of the phenotypic transformation of VSMCs may provide a theoretical basis for the development and treatment of vascular diseases.

Previous studies have focused on the molecular and biological mechanisms of VSMC phenotype transformation, including investigating microRNAs and transcription factors $(7,8)$. GC-binding factor 2 (GCF2) is a transcriptional repressors that binds to gene promoters rich in $\mathrm{GC}$ and inhibits transcription (9). As a transcriptional repressor, the main function of GCF2 is to inhibit or downregulate the transcription of target genes. The amino acid residues at positions 429-528 of the peptide chain are important for the direct binding of GCF2 to GC sequences in the promoter of genes, including endothelial growth factor receptor (EGFR) (10). In a number of signal transduction pathways, GCF2 is a protein that binds to related proteins, such as dishevelled protein, and is involved in signal transduction, cell proliferation, apoptosis and cell cycle regulation $(11,12)$. There are a number of cell cycle regulatory proteins involved in maintaining genomic integrity and stability in normal cells. However, aberrant cell cycle regulation leads to abnormal gene expression levels, which in turn 
leads to uncontrolled proliferation and apoptosis, ultimately leading to tumor growth $(13,14)$. At the same time, dysplasia of VSMCs is regulated by multiple pathways, including the PI3K/AKT signaling pathway $(15,16)$.

The PI3K/AKT signal transduction pathway is an important signaling pathway for survival in vivo (17). PI3K is a class of phosphorylated inositol phospholipid 3 hydroxyl kinase with lipid and protein kinase activities. AKT is an important downstream target in the PI3K signal transduction pathway. AKT has serine/threonine kinase activity, which is activated by the phosphorylation of AKT by PI3K, further activating other downstream factors (18).

At present, the role of GCF2 in the proliferation of VSMCs is not well documented. In the present study, small interfering RNA (siRNA) technology was used to reduce the expression of GCF2 and investigate the role of GCF2 in vascular smooth muscle function. Therefore, this present study provides mechanistic insight to facilitate the development of therapies for the prevention, diagnosis and treatment of cardiovascular disease.

\section{Materials and methods}

Isolation and culture of VSMCs from the C57/BL6-mouse aorta. The adult male C57/BL6 mice $(n=6 ; 8-10$ weeks of age; weight, 18-22 g) used in this study were obtained from the Shanghai Laboratory Animal Co., Ltd. The animals were housed with food and water available ad libitum and kept at a controlled room temperature $\left(22 \pm 2^{\circ} \mathrm{C}\right)$ and humidity $(60-80 \%)$ under a 12/12 h light/dark cycle. C57/BL6 mice were weighed and anesthetized by intraperitoneal injection of $10 \%$ chloral hydrate $(300 \mathrm{mg} / \mathrm{kg})$, and peritonitis was not observed in any of the mice. After anesthesia, a continuous flow of $\mathrm{CO}_{2}$ was maintained using a flow meter unit for 3-5 min at the flow rate of $2 \mathrm{l} / \mathrm{min}$ for sacrifice. The mice were soaked in $75 \%$ ethanol for $2 \mathrm{~min}$ at room temperature for disinfection and affixed to a wooden board. The mouse thoracic cavity and abdominal cavity were opened, the heart removed and the whole thoracic aorta and abdominal aorta was fully exposed along the aorta. A $5 \mathrm{ml}$ syringe was inserted through the left ventricle and the aorta was flushed with PBS buffer, removed and placed in a Petri dish filled with PBS buffer. The aortic intima and adventitia were cut into approximately 1-2 $\mathrm{mm}^{2}$ sections with ophthalmic scissors. Sections were uniformly arranged at a density of 4-7 pieces $/ \mathrm{cm}^{2}$ in culture plates and $5 \mathrm{ml} \mathrm{DMEM} \mathrm{(Corning,}$ Inc.) containing $100 \mathrm{U} / \mathrm{ml}$ penicillin, $100 \mu \mathrm{g} / \mathrm{ml}$ streptomycin

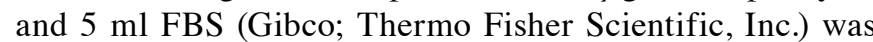
added. The sections were cultured in an incubator at $37^{\circ} \mathrm{C}$ with $5 \% \mathrm{CO}_{2}$, the medium was changed every 3 days. After 8 days, the tissue sections were removed with sterile forceps. The cells were transferred to another culture dish, and allowed to grow for 10 days, until they covered an area of $25 \mathrm{~cm}^{2}$. Then, the cells were subcultured four times. Subsequently, the cells were digested with trypsin and collected. For experiments, cells at passage number 4-10 were used.

Immunofluorescence staining. Cells, at a density of $2 \times 10^{5}$ cells/well, were seeded in a 24 -well culture plate with built-in cover slips. After $24 \mathrm{~h}$, when the cells adhered naturally and reached a confluence of $80 \%$, the culture medium was discarded and the cover slips were removed. The cover slips were washed three times for 3 min with PBS and then fixed for $30 \mathrm{~min}$ with $40 \mathrm{~g} / 1$ paraformaldehyde at room temperature and washed three times for 3 min with PBS. The cells were incubated for $30 \mathrm{~min}$ with $3 \mathrm{~g} / \mathrm{l}$ of Triton-X-100, washed with PBS three times for $3 \mathrm{~min}$ and blocked with $250 \mu \mathrm{l} \mathrm{FBS}$ at room temperature for $30 \mathrm{~min}$. The cells were incubated overnight with goat anti-mouse $\alpha$-SM actin (1:100; cat. no. a5228; Sigma-Aldrich; Merck KGaA) at $4^{\circ} \mathrm{C}$, washed with TBS three times for $15 \mathrm{~min}$, incubated with a rabbit anti-sheep secondary antibody conjugated to tetraethyl rhodamine isothiocyanate (1:100; cat. no. sc-215957; Santa Cruz Biotechnology, Inc) at room temperature for $1 \mathrm{~h}$ and washed with PBS for three times for $3 \mathrm{~min}$. DAPI $(1 \mu \mathrm{g} / \mathrm{ml})$ was added to the coverslips and incubated while being protected from light for $10 \mathrm{~min}$ at room temperature to stain the nuclei. Images were captured using a fluorescent microscope (magnification, x400).

Transfections and IGF-1 treatment. Cells, at a density of $2 \times 10^{5}$ cells/well, were seeded in 24-well culture plate and $500 \mu \mathrm{l}$ of antibiotic-free medium was added to each well. A confluency of $80 \%$ was reached prior to transfection. For each well, a reaction containing $1 \mu 1$ Lipofectamine ${ }^{\circledR} 2000$ (Thermo Fisher Scientific, Inc.) was diluted with $50 \mu$ Opti-MEM $^{\mathrm{TM}}$ Reduced Serum Medium (Gibco; Thermo Fisher Scientific, Inc.) was prepared, gently mixed and incubated for $5 \mathrm{~min}$ at room temperature. A second reaction, containing $2 \mu 1$ GCF2-siRNA (Shanghai GenePharma Co., Ltd.) diluted with $50 \mu 1$ of Opti-MEM ${ }^{\mathrm{TM}}$ Reduced Serum Medium was prepared, mixed gently and incubated for $5 \mathrm{~min}$ at room temperature. The diluted Lipofectamine ${ }^{\circledR} 2000$ was gently mixed with the diluted GCF2-siRNA and allowed to stand at room temperature for a further $20 \mathrm{~min}$ to form a GCF2-siRNA-transfection reagent mixture. The GCF2-siRNA-transfection reagent mixture was added to the culture medium containing the cells, mixed and placed in an incubator at $37^{\circ} \mathrm{C}$ with $5 \% \mathrm{CO}_{2}$ for $48 \mathrm{~h}$. All experiments were performed according to the manufacturer's protocol (Thermo Fisher Scientific, Inc.). The sequences of the siRNAs used in the present study were as follows: GCF2-siRNA sense, 5'-GGAAAUCAAGGACUCUCUAGC AGAA-3' and antisense, 5'-UUCUGCUAGAGAGUCCUU GAUUUCC-3'; negative control-siRNA (siNC) sense, 5'-UUC UCCGAACGUGUCACGUTT-3' and antisense, 5'-ACGUGA CACGUUCGGAGAATT-3'. In addition, to further investigate the mechanism of GCF2 on VSMCs, cells were treated with IGF-1 (120 ng/ml) for $48 \mathrm{~h}$. Subsequently, cell viability, migration and invasion were assessed. The cells were divided into six groups: i) control, normal cell group; ii) siNC, cells transfected with siNC; iii) siGCF2, cells transfected with siGCF2; iv) IGF-1, cells treated with $120 \mathrm{ng} / \mathrm{ml}$ IGF-1; v) siNC + IGF-1, cells transfected with siNC and treated with $120 \mathrm{ng} / \mathrm{ml} \mathrm{IGF-1;}$ and vi) siGCF2 + IGF-1, cells transfected with siGCF2 and treated with $120 \mathrm{ng} / \mathrm{ml} \mathrm{IGF-1.}$

Cell Counting Kit-8 (CCK-8). Transfected cells, at a density of $3 \times 10^{3}$ cells/well, were seeded into 96-well plates and cultured for 24,48 or $72 \mathrm{~h}$ in an incubator with $5 \% \mathrm{CO}_{2}$ at $37^{\circ} \mathrm{C}$. CCK-8 (Nanjing Jiancheng Bioengineering Institute) and serum-free DMEM at a ratio of 1:10 were mixed and $100 \mu \mathrm{l}$ was added to each well and incubated at $37^{\circ} \mathrm{C}$ with $5 \% \mathrm{CO}_{2}$ for $1 \mathrm{~h}$. A microplate reader (Bio-Rad Laboratories, Inc.) was used to 
determine the optical density of each well at an absorbance of $450 \mathrm{~nm}$. Cell viability was determined using the CCK-8 Kit according to the manufacturer's protocol.

Wound healing assay. After the VSMCs had been transfected for $48 \mathrm{~h}$, a straight gap was created using a $200 \mu \mathrm{l}$ sterile tip in the middle of the well. The cells were washed twice with DMEM to smooth the edges of the scratch and remove floating cells. After incubation at $37^{\circ} \mathrm{C}$ for 0 or $48 \mathrm{~h}$, the cells were observed under a florescent microscope (Keyence Corporation; magnification, x100) and the distance of migration was determined. Images were captured using Image-Pro Plus Analysis software (version 6.0; Media Cybernetics, Inc.).

Transwell. Transwell chambers with $8.0 \mu \mathrm{m}$ pores (Corning, Inc.) were placed on a 24 -well plate with a $50 \mu$ l layer of Matrigel (BD Biosciences). The Matrigel was diluted 1:8, coated on the upper chamber of the bottom membrane of the Transwell chamber and placed in an incubator at $37^{\circ} \mathrm{C}$ for $30 \mathrm{~min}$ to polymerize the Matrigel into a gel. A cell suspension, at a density of $1 \times 10^{5}$ cells $/ 200 \mu 1$, was added to the upper layer of the Transwell chamber and $600 \mu \mathrm{l}$ of $20 \%$ FBS was added to the lower chamber. After incubation for $48 \mathrm{~h}$ at $37^{\circ} \mathrm{C}$, cells that had not invaded the Transwell chamber were gently removed with a cotton swab. The chamber was air-dried and fixed in $4 \%$ paraformaldehyde for $15 \mathrm{~min}$ and stained with $0.1 \%$ crystal violet for $20 \mathrm{~min}$. Cells from five randomly selected fields were observed under a florescent microscope (magnification, x200) and counted.

Apoptosis. VSMCs, at a density of $2 \times 10^{5}$ cells/well, were seeded in 6-well plates. After the cells had been treated, the supernatant was collected in a $15 \mathrm{ml}$ centrifuge tube and the culture flask was gently washed once using $2 \mathrm{ml}$ PBS. The cells were digested with $1 \mathrm{ml}$ trypsin without EDTA, gently shaken, and the trypsin was aspirated immediately. The mixture was left at room temperature for $1 \mathrm{~min}$, after which DMEM containing $10 \%$ FBS was added to terminate the digestion. The cells were centrifuged at $1,000 \mathrm{x}$ g for $3 \mathrm{~min}$ at $4^{\circ} \mathrm{C}$ and the supernatant was removed. The cells were washed twice with pre-cooled PBS and resuspended in $1 \mathrm{X}$ Annexin $\mathrm{V}$ binding buffer. According to the Annexin-V-FITC apoptosis kit (BioVision, Inc.), cells were collected and stained with Annexin V-FITC and propidium iodide (PI) for $15 \mathrm{~min}$ at room temperature. Cells were counted by flow cytometry (FACS Calibur ${ }^{\mathrm{TM}}$; Becton, Dickinson and Company) and analyzed using FlowJo (version 10.0; FlowJo, LLC). Flow cytometry scatter diagrams showed that living cells were in the lower left quadrant, necrotic cells were in the left upper quadrant, advanced apoptotic cells were in the upper right quadrant, and early apoptotic cells were in the lower right quadrant.

Flow cytometry cell cycle analysis. Cell cycle analysis was performed using flow-cytometry. For fixation, $5 \times 10^{5}$ cells were incubated with $70 \%$ ice-cold ethanol at $-20^{\circ} \mathrm{C}$ overnight. The next day, the fixed cells were centrifuged at $1,200 \mathrm{x} \mathrm{g}$ for $1 \mathrm{~min}$ at $4^{\circ} \mathrm{C}$ and washed twice with PBS. The cells were resuspended in $200 \mu \mathrm{l} \mathrm{RNaseA}(1 \mathrm{mg} / \mathrm{ml})$ for $10 \mathrm{~min}$ at $37^{\circ} \mathrm{C}, 300 \mu \mathrm{l}$ PI (BioVision, Inc.) was added, and the cells were incubated at room temperature for $20 \mathrm{~min}$ to stain the DNA. The cells were protected from light during the staining procedure. The cells were analyzed for cellular DNA content using Mod Fit LT software V2.0 (Becton Dickinson and Company) with a FACScan flow cytometer (Becton, Dickinson, and Company).

Reverse transcription-quantitative $(R T-q) P C R$. RNA was extracted using TRIzol ${ }^{\circledR}$ (Invitrogen; Thermo Fisher Scientific, Inc.), according to the manufacturer's protocol. For reverse transcription, $1 \mu \mathrm{g}$ RNA was to generate cDNA according to the SuperScript III CellsDirect cDNA Synthesis kit (Thermo Fisher Scientific, Inc.). The reaction conditions were as follows: $42^{\circ} \mathrm{C}$ for $15 \mathrm{~min}$ and $80^{\circ} \mathrm{C}$ for $15 \mathrm{sec}$. SYBR Green PCR Master Mix (Roche Diagnostics) was used to conduct the qPCR experiments using Opticon RT-PCR Detection System (ABI 7500; Life Technologies; Thermo Fisher Scientific, Inc.). The qPCR conditions were as follows: $95^{\circ} \mathrm{C}$ for $10 \mathrm{~min}$, followed by 40 cycles of $94^{\circ} \mathrm{C}$ for $15 \mathrm{sec}, 60^{\circ} \mathrm{C}$ for $1 \mathrm{~min}$ and $60^{\circ} \mathrm{C}$ for $1 \mathrm{~min}$. The expression levels of the genes were analyzed using the $2^{-\Delta \Delta \mathrm{Cq}}$ method (19). GAPDH expression was used for normalization. The primer sequences of GCF2, Bcl-2, Bax, Cleaved caspase-3, cyclin E, CDK2, P21 and GAPDH are listed in Table I.

Western blot analysis. Total proteins were extracted using RIPA lysis buffer (Cell Signaling Technology, Inc.). A bicinchoninic acid protein assay kit (Pierce; Thermo Fisher Scientific, Inc.) was used to determine protein concentration. Concentration were adjusted to $6 \mu \mathrm{g} / \mu \mathrm{l}$ using $1 \mathrm{X}$ loading buffer and diethyl pyrocarbonate-treated water. Samples (30 $\mu \mathrm{g} /$ lane) were separated using 10\% SDS-PAGE gels, which were then transferred onto a PVDF membrane. After blocking in 5\% non-fat milk in PBS-0.1\% Tween-20 for $1 \mathrm{~h}$ at room temperature, the membrane was probed with primary antibodies overnight at $4^{\circ} \mathrm{C}$. Membranes were then incubated with horseradish peroxidase-conjugated secondary antibodies, including goat anti-mouse (1:2,000; cat. no. sc-516102; Santa Cruz Biotechnology, Inc.) and goat anti-rabbit (1:2,000; cat. no. sc-2357; Santa Cruz Biotechnology, Inc.) at room temperature for $2 \mathrm{~h}$. The EZ-ECL kit (Biological Industries) was used to visualize the protein bands and densitometric analysis was carried out using ImageJ (version 5.0; National Institutes of Health). The primary antibodies utilized included anti-GAPDH (mouse; 1:1,000; cat. no. LS-B1625; LifeSpan BioSciences, Inc.), anti-cleaved caspase-3 (rabbit; 1:1,000; cat. no. ab13847; Abcam), anti-Bax (rabbit; 1:1,000; cat. no. ab32503; Abcam), anti-Bcl-2 (rabbit; 1:1,000; cat. no. ab32124; Abcam), anti-CDK2 (rabbit; 1:1,000; cat. no. ab32147; Abcam), anti-P21 (rabbit; 1:1,000; cat. no. ab109520; Abcam), anti-AKT (rabbit; 1:1,000; cat. no. ab8805; Abcam), anti-p-AKT (rabbit; 1:1,000; cat. no. ab38449; Abcam), anti-p-PI3K (rabbit; 1:1,000; cat. no. ab182651; Abcam) and anti-PI3K (rabbit; 1:1,000; cat. no. 4257; Cell Signaling Technology, Inc.).

Statistical analysis. All data are expressed as the mean \pm SEM. The differences between the experimental groups were compared by Student's t-test and one-way ANOVA followed by Dunnett's post hoc test. Data analysis was performed using the statistical software Prism 6 (GraphPad Software, Inc.). $\mathrm{P}<0.05$ was considered to indicate a statistically significant difference. All experiments were independently repeated three times. 
Table I. Primers for reverse transcription-quantitative PCR.

\begin{tabular}{lll}
\hline Genes & \multicolumn{1}{c}{ Forward $\left(5^{\prime}-3 '\right)$} & \multicolumn{1}{c}{ Reverse $\left(5^{\prime}-3^{\prime}\right)$} \\
\hline GCF2 & TGAAAGGGAAAAACACGCC & TCATTTTTCACCTCCACTTCAC \\
Bcl-2 & CGACTTTGCAGAGATGTGCA & ATGCCGGTTCAGGTACTCAG \\
Cleaved caspase-3 & AGCAGCTTTGTGTGTGTGATTAA & AGTTTCGGCTTTCCAGTCAGAC \\
Cyclin E & GTTACAGATGGCGCTTGCTC & AGCCAGGACACAATGGTCAGCAGT \\
CDK2 & TTGGAGTCCCTGTCCGAACT & CGGGTCACCATTTCAGCAAAG \\
p21 & CAAAGTGTGCCGTTGTCTCTT & TCAAAGTTCCACCGTTCTCG \\
GAPDH & CATCACCATCTTCCAGGAGGG & TGACCTTGCCCACAGCCTTG
\end{tabular}

GCF2, GC binding factor 2 .
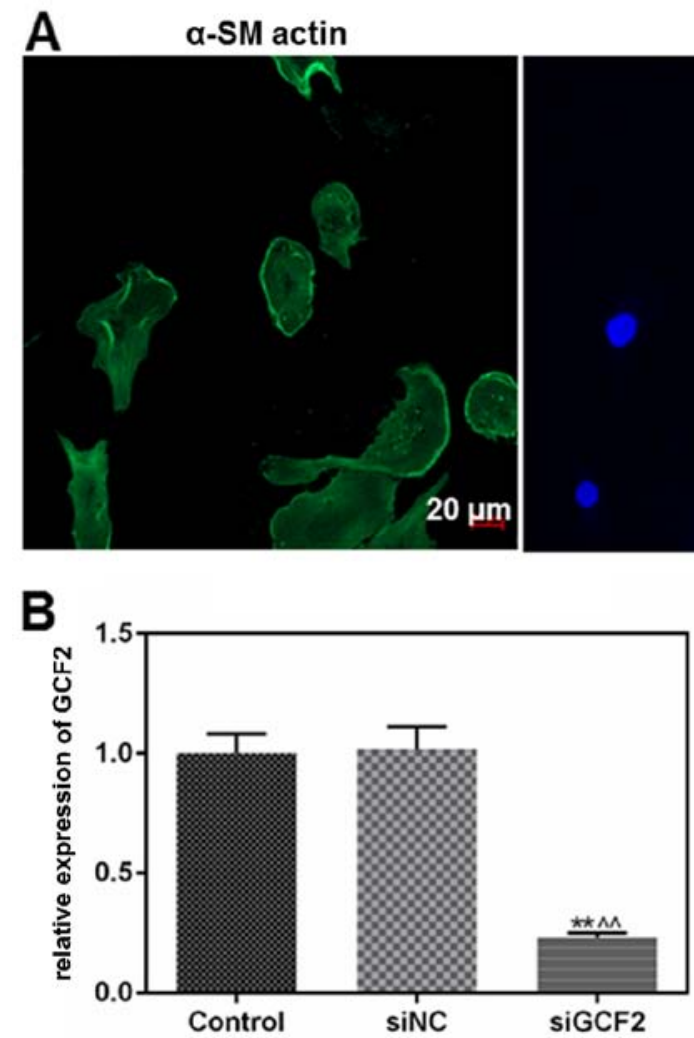

DAPI

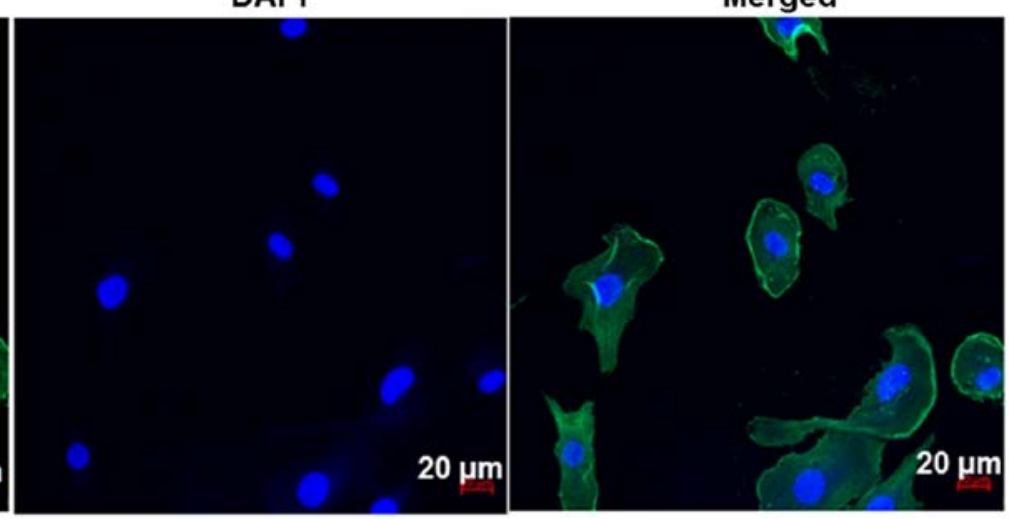

C

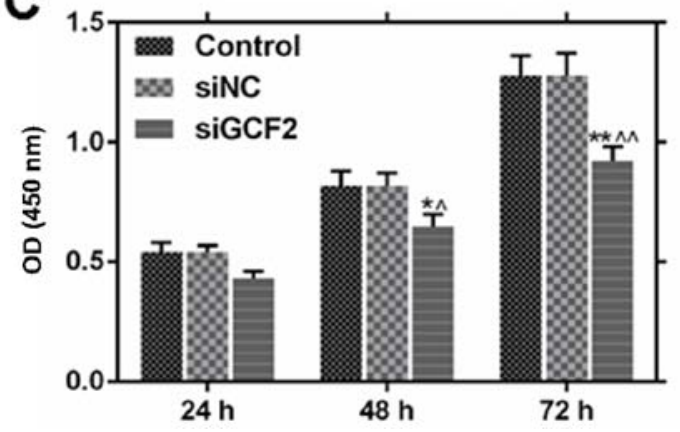

Figure 1. Viability was reduced by siGCF2. (A) $\alpha$-SM actin was detected by immunofluorescence. (B) The transfection efficiency of siGCF2 was determined by reverse transcription-quantitative PCR. (C) The viability of cells was determined using the Cell Counting Kit-8. ${ }^{*} \mathrm{P}<0.05$, ${ }^{* *} \mathrm{P}<0.01$ vs. respective control; ${ }^{\wedge} \mathrm{P}<0.05,{ }^{\wedge} \mathrm{P}<0.01$ vs. respective siNC. GCF2, GC binding factor 2; siGCF2, small interfering RNA targeting GCF2; siNC, non-targeting small interfering RNA; $\alpha$-SM, $\alpha$-smooth muscle actin; OD, optical density.

\section{Results}

Identification of VSMCs by immunofluorescence staining and analysis of cell viability of following treatment with siGCF2. To explore the effect of GCF2 on migration and invasion, primary VSMCs were isolated from the mouse aorta and siGCF2 was used to reduce the expression of GCF2. The cells were stained for $\alpha$-SM actin and immunofluorescence analysis was used to determine whether cell function was restored. Immunofluorescence analysis showed $\alpha-\mathrm{SM}$ actin staining in the cytoplasm, and that the morphology and the nuclear outline were clear. $\alpha$-SM actin had a positive expression, indicating that the cells were VSMCs (Fig. 1A). GCF2 expression was successfully reduced by transfection with siGCF2 at the mRNA level (Fig. 1B). Cell viability was inhibited by transfection with siGCF2 at 48 and $72 \mathrm{~h}$ (Fig. 1C).

Migration and invasion are inhibited by siGCF2. Wound healing and Transwell assays were used to determine the migration and invasion ability of cells to investigate the function of GCF2 in VSMCs. Migration (Fig. 2A) and invasion (Fig. 2B) were inhibited by siGCF2 at $48 \mathrm{~h}$ post transfection.

Apoptosis is increased following transfection with siGCF2. Apoptosis is a physiological process that is important for cell survival. In this present study, apoptosis was analyzed 

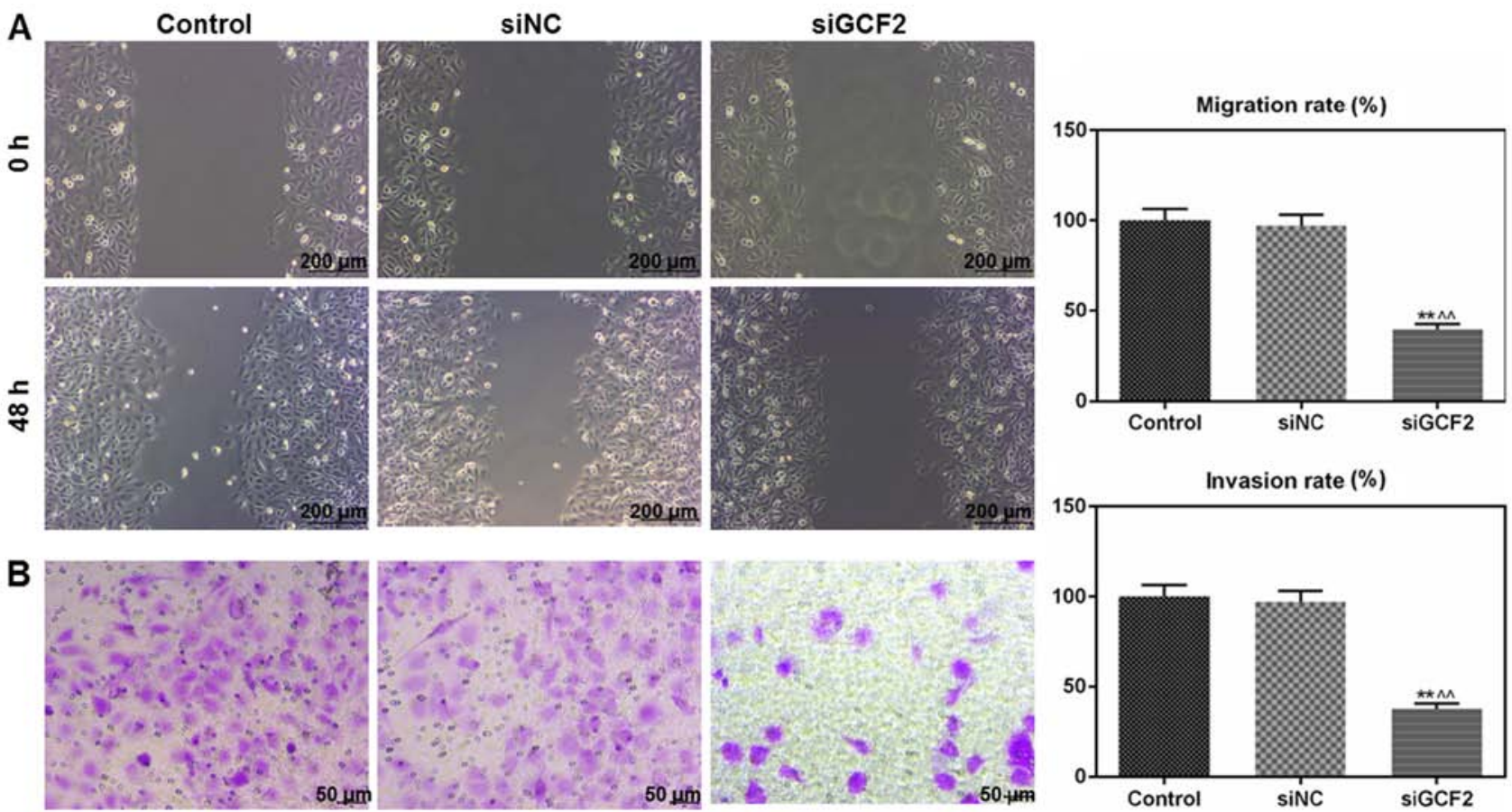

Figure 2. Migration and invasion were inhibited by siGCF2. (A) The ability of cells to migrate was determined using a wound healing assay (magnification; x200). (B) Invasion was analyzed using a Transwell assay (magnification; $\mathrm{x} 200$ ). ${ }^{* * *} \mathrm{P}<0.01 \mathrm{vs.} \mathrm{respective} \mathrm{control;}{ }^{\wedge} \mathrm{P}<0.01 \mathrm{vs}$. respective siNC. GCF2, GC binding factor 2; siGCF2, small interfering RNA targeting GCF2; siNC, non-targeting small interfering RNA.
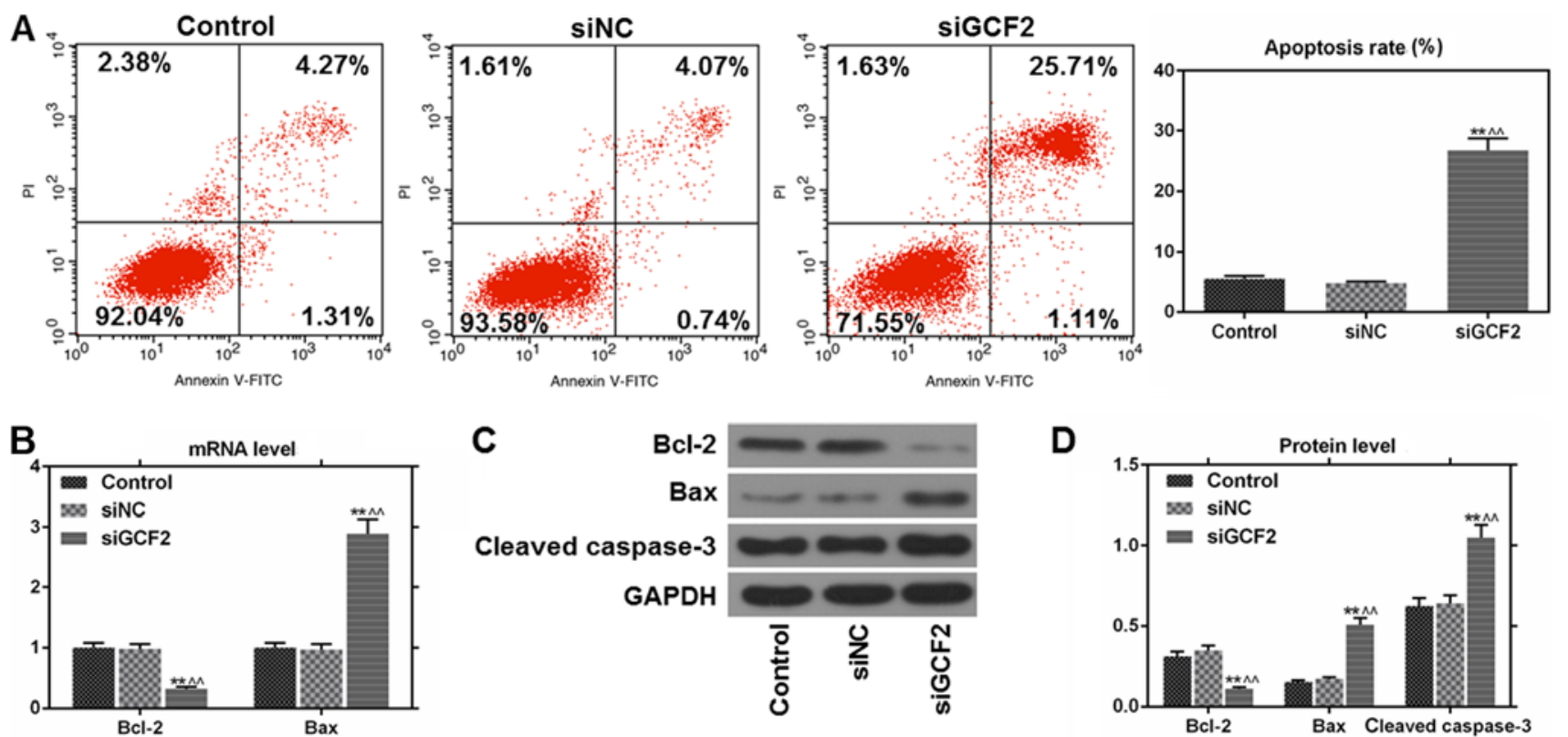

Figure 3. Apoptosis was increased by siGCF2. (A) The level of apoptosis was analyzed by flow cytometry. (B) The mRNA levels of Bcl-2 and Bax were determined by reverse transcription-quantitative PCR. (C) The protein levels of Bcl-2, Bax and cleaved caspase-3 were determined by western blot analysis. (D) Quantification of western blot analysis using GAPDH for normalization. ${ }^{* *} \mathrm{P}<0.01 \mathrm{vs}$. respective control; ${ }^{\wedge} \mathrm{P}<0.01 \mathrm{vs}$. respective siNC. GCF2, GC binding factor 2; siGCF2, small interfering RNA targeting GCF2; siNC, non-targeting small interfering RNA.

by flow cytometry. The level of apoptosis was found to be higher in cells transfected with siGCF2 compared to control cells and cells transfected with a non-targeting siRNA control (siNC) (Fig. 3A). Additionally, the mRNA and protein expression levels of apoptosis-related factors were determined by RT-qPCR and western blot analysis. The expression of Bcl-2 was downregulated, while the expression of Bax and cleaved caspase-3 was upregulated in cells transfected with siGCF2 at the mRNA (Fig. 3B) and/or protein level (Fig. 3C and D).

Cell cycle arrest is induced by siGCF2. Cell cycle analysis was conducted using flow cytometry. The results showed that cell cycle arrest in the $G_{0} / G_{1}$ phase of the cell cycle was induced by siGCF2 (Fig. 4A). Additionally, the mRNA and protein 

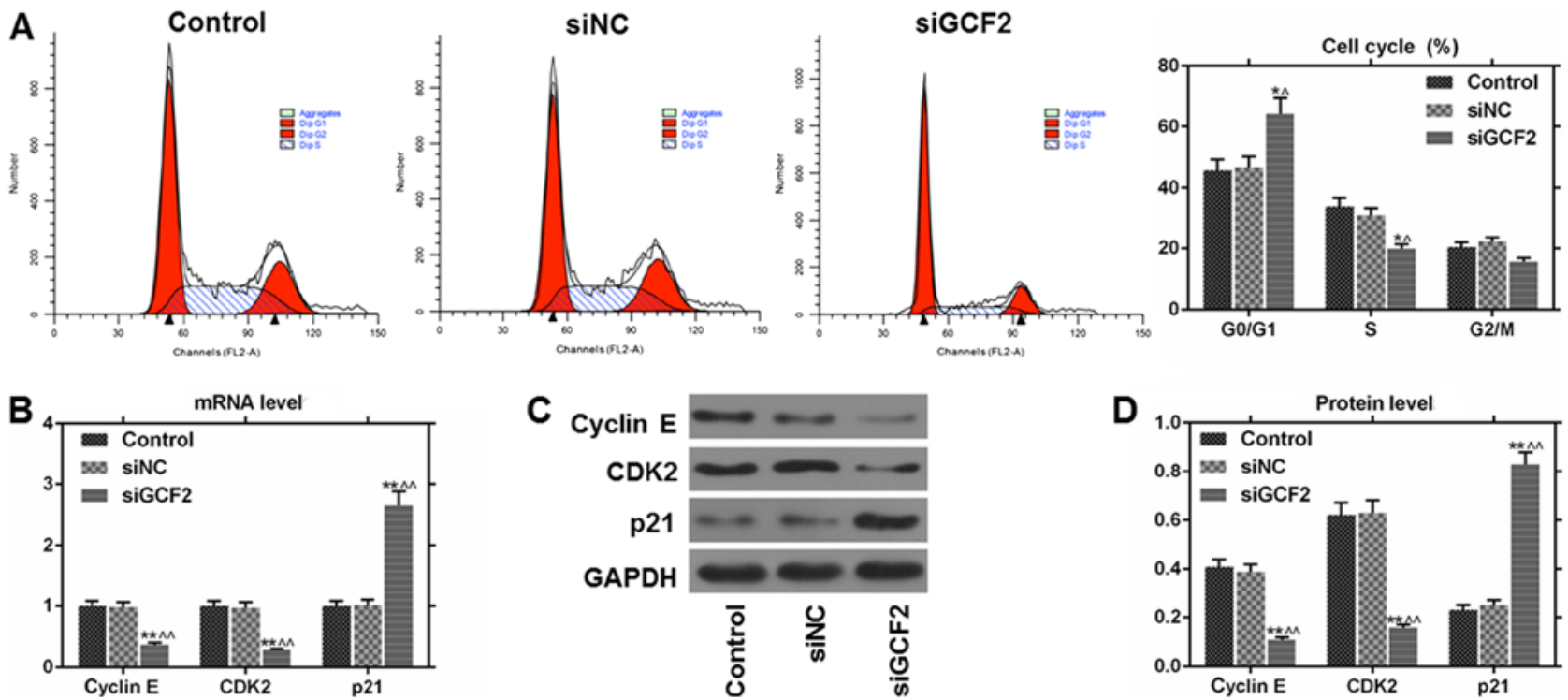

Figure 4. Cell cycle arrest in the $\mathrm{G}_{0} / \mathrm{G}_{1}$ phase was induced by siGCF2. (A) Cell cycle progression was analyzed by flow cytometry. (B) The mRNA levels of cyclin E, CDK2 and p21 were determined by reverse transcription-quantitative PCR. (C) The protein levels of cyclin E, CDK2 and p21 were determined by western blot analysis. (D) Quantification of western blot analysis using GAPDH for normalization. ${ }^{\text {}} \mathrm{P}<0.05,{ }^{* *} \mathrm{P}<0.01$ vs. respective control; ${ }^{\wedge} \mathrm{P}<0.05$, ${ }^{\wedge} \mathrm{P}<0.01$ vs. respective siNC. GCF2, GC binding factor 2; siGCF2, small interfering RNA targeting GCF2; siNC, non-targeting small interfering RNA.

expression levels of cell cycle regulators were determined by RT-qPCR and western blot analysis. The levels of cyclin E and CDK2 were higher in control cells and those transfected with siNC than in cells transfected with siGCF2. By contrast, the expression of p21 was lower in control cells and those transfected with siNC than in cells transfected with in siGCF2 at the mRNA (Fig. 4B) and protein level (Fig. 4C and D).

Phosphorylation of PI3K and AKT is downregulated by $s i G C F 2$. As the PI3K/AKT signaling pathway is important in the development of VSMCs, western blot analysis was used to determine the protein levels of phosphorylated PI3K and AKT. The levels of phosphorylated PI3K and AKT were reduced by siGCF2 (Fig. 5).

Inhibitory effects of siGCF2 on cell viability, migration and invasion were reversed by insulin-like growth factor 1 $(I G F-1)$. In order to investigate the role of the PI3K/AKT signaling pathway in VSMCs, cells were exposed to IGF-1 $(120 \mathrm{ng} / \mathrm{ml})$, which is a specific agonist of AKT, in combination with siRNA. The levels of cell viability, migration and invasion were analyzed by CCK- 8 , wound healing and Transwell assays, respectively. The results indicated that cell viability (Fig. 6A), migration (Fig. 6B) and invasion (Fig. 6C) were reduced in cells exposed to IGF-1 in combination with siGCF2. Representative images of migration and invasion are shown in Fig. 6D and E, respectively.

\section{Discussion}

GCF2, also known as leucine-rich repeat flightless-interacting protein, is an inhibitory transcription factor that can bind $\mathrm{GC}$ in the gene promoter sequences of platelet-derived growth factor A, EGFR and tumor necrosis factor $\alpha$ to inhibit transcription $(9,20,21)$. In this present study, it was found that invasion and migration were inhibited in cells transfected

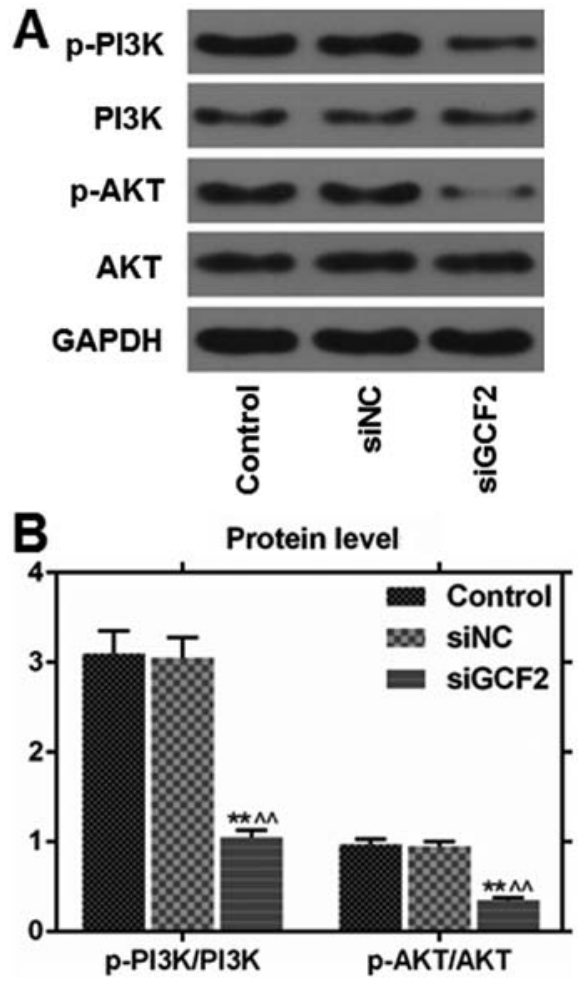

Figure 5. Phosphorylation of PI3K and AKT was reduced by siGCF2. (A) The levels of phosphorylated PI3K and AKT were determined by western blot analysis. (B) Quantification of western blot analysis using GAPDH for normalization. ${ }^{* *} \mathrm{P}<0.01$ vs. respective control; ${ }^{\wedge} \mathrm{P}<0.01$ vs. respective siNC. GCF2, GC binding factor 2; siGCF2, small interfering RNA targeting GCF2; siNC, non-targeting small interfering RNA; p-PI3K, phosphorylated PI3K; p-AKT, phosphorylated AKT.

with siGCF2, while apoptosis and cell cycle arrest in the $\mathrm{G}_{0} / \mathrm{G}_{1}$ phase were induced by siGCF 2 in VSMCs. These effects may be associated with the PI3K/AKT signaling pathway. 
A

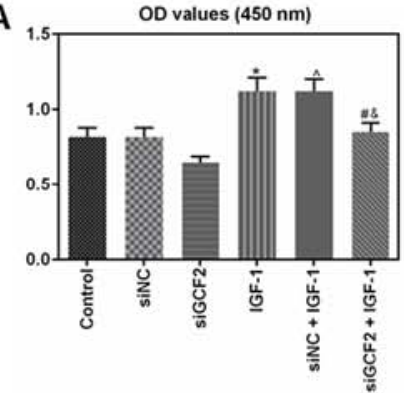

B

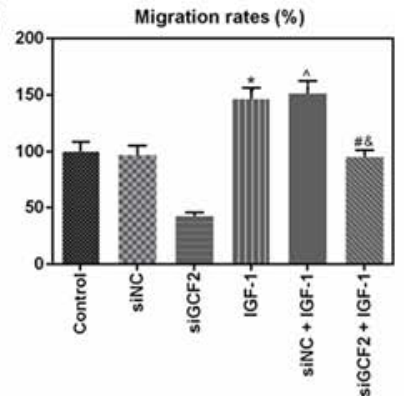

C

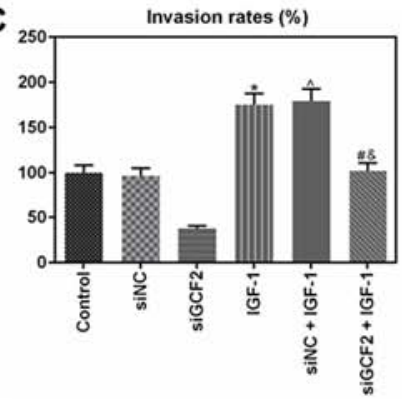

D
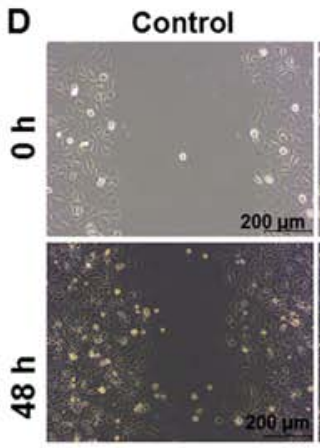

E

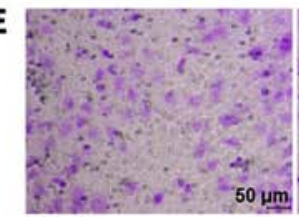

siNC
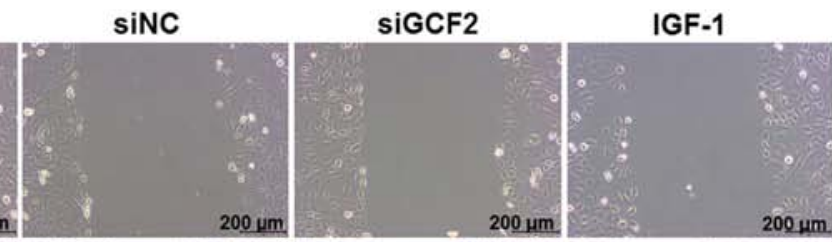

siNC+IGF-1
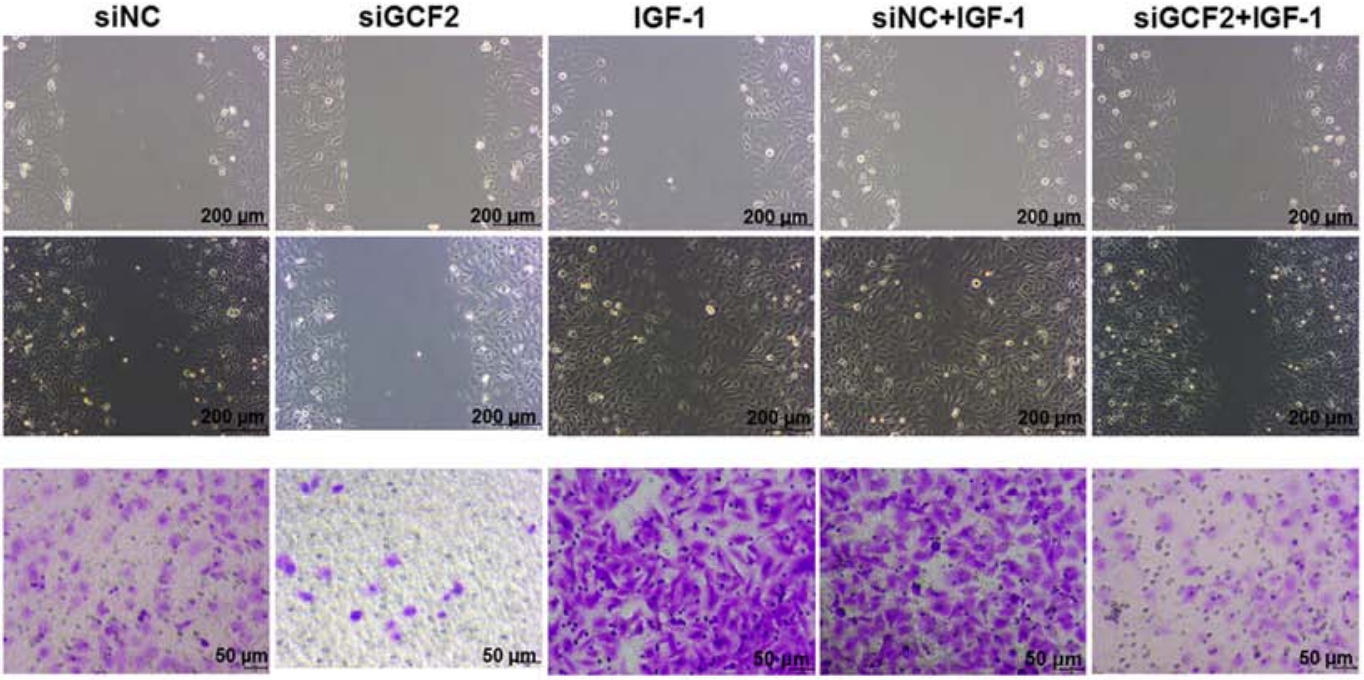

Figure 6. Reduced viability, migration and invasion caused by siGCF2 were reversed by IGF-1. (A) Viability was determined using the Cell Counting Kit-8 assay. (B) The ability of cells to migrate was determined using a wound healing assay. (C) Invasion was analyzed using a Transwell migration assay. (D) Representative images of migration (magnification, $\mathrm{x} 200$ ) and (E) invasion (magnification, $\mathrm{x} 200$ ). ${ }^{*} \mathrm{P}<0.05$ vs. respective control; ${ }^{\wedge} \mathrm{P}<0.05$ vs. respective siNC; ${ }^{*} \mathrm{P}<0.05$ vs. respective siGCF2; ${ }^{\star} \mathrm{P}<0.05$ vs. respective siNC + IGF-1. GCF2, GC binding factor 2 ; siGCF2, small interfering RNA targeting GCF2; siNC, non-targeting small interfering RNA; IGF-1, insulin-like growth factor 1; OD, optical density.

In order to study the molecular basis of the abnormal proliferation of VSMCs, VSMCs were stripped from the aortas of mice. To confirm whether VSMCs from mice could be used as model, cells were stained for $\alpha$-SM actin, which has an important role in maintaining vascular tone and homeostasis. The results showed that $\alpha$-SM actin was positively expressed, suggesting that VSMCs were successfully obtained and could be used in subsequent experiments.

RNA interference technology is an effective means to study gene function, and can be used to efficiently and specifically reduce the expression of target genes in vitro (22). siGCF2 sequences targeting GCF2 were designed and transfected into VSMCs. The results showed that siGCF2 effectively downregulated GCF2 mRNA expression in VSMCs, therefore facilitating the investigation of the effect of GCF2 on the migration and invasion of VSMCs.

It is thought that the phenotypic transition of VSMCs is an important pathophysiological basis for neointimal hyperplasia after vascular injury, and that this can ultimately lead to stenosis of the lumen (23). In a pathological case, a synthetic phenotype characterized by proliferation and migration is exhibited (24). A previous study demonstrated that smooth muscle cell proliferation is inhibited by GCF2 (20). In the present study, it was found that cell viability, migration and invasion are inhibited by siGCF2. Therefore, GCF2 may enhance the proliferation of VSMCs.
Proliferation and apoptosis are often linked (25); apoptosis plays an important role in maintaining the balance between the production of new cells and cell death in tissues (26). In the present study, the analysis of apoptosis showed that the level of apoptosis and the levels of cleaved caspase 3 and Bax were significantly increased. By contrast, the expression level of the anti-apoptotic protein $\mathrm{Bcl}-2$ was downregulated after GCF2 knockdown. These findings indicated that GCF2 knockdown may inhibit the proliferation of VSMCs by regulating the expression of apoptosis-associated proteins. In the present study, the mechanism by which siGCF2 promotes apoptosis was not explored further. The expression levels of forkhead box protein O3, which is a key regulator of the PI3K/Akt signaling pathway, and the pro-apoptotic protein Bcl-2-like protein 11, were not investigated in this present study. These factors will be assessed in future studies.

Abnormal cell proliferation reflects misregulation of the cell cycle (27). The results of the present study showed that downregulation of GCF2 expression caused VSMC proliferation to be blocked, suggesting misregulation of the cell cycle. The proportion of VSMCs in the $\mathrm{G}_{0} / \mathrm{G}_{1}$ phase of the cell cycle was significantly higher in siGCF2 group than in the control group, indicating that downregulation of GCF2 expression caused cell cycle arrest in the $\mathrm{G}_{1}$ phase. The cell cycle is regulated by cyclins and CDKs; together they form 
cyclin-CDK complexes that regulate cell cycle transitions and DNA synthesis. Cyclin E-CDK2 is important during the $\mathrm{G}_{1}$ to $\mathrm{S}$ phase transition $(28,29)$. p21 is a CDK inhibitor with broad kinase inhibitory activity. p21 has an inhibitory effect on proliferation (30). In the present study, it was found that the downregulation of GCF2 expression led to a significant reduction in the expression of cyclin $\mathrm{E}$ and $\mathrm{CDK} 2$, while p21 expression was significantly increased. Based on the findings of the present study, it is speculated that the inhibitory effect of GCF2 downregulation on the proliferation of VSMCs could be a result of changes to the regulation of the cell cycle.

The PI3K/AKT signaling pathway is important in VSMC proliferation and migration $(15,31)$. Previous studies have reported that PI3K/AKT activation is important in promoting the migration of cultured VSMCs; however, this effect is increased by AKT depletion $(32,33)$. Another study also found that PI3K/AKT inhibition blocked serum-stimulated VSMC lamellipodia formation (15). In the present study, the phosphorylation levels of PI3K and AKT were found to be reduced by siGCF2. IGF-1 reversed the inhibitory effect of siGCF2 on viability, migration and invasion in VSMCs. These results suggested that the inhibition of VSMC proliferation observed following the transfection of cells with siGCF2 may be mediated by the PI3K/AKT signaling pathway.

The effect of GCF2 downregulation on VSMC proliferation, migration and invasion is only supported by in vitro experiments in the present study. Therefore, such an effect should be further investigated by conducting in vivo experiments. Additionally, the underlying mechanism of the regulation of VSMCs by siGCF2 is still unclear and requires further investigation.

In conclusion, a novel role for GCF2 in VSMC proliferation in vitro has been identified. The inhibition of VSMC proliferation and migration by siGCF 2 was mediated by the PI3K/AKT signaling pathway. The findings of the present study suggest that GCF2 may be used as a novel therapeutic agent for vascular restenosis.

\section{Acknowledgements}

Not applicable.

\section{Funding}

No funding was received.

\section{Availability of data and materials}

The data sets generated and analyzed during the present study are available from the corresponding author on reasonable request.

\section{Authors' contributions}

YM and YR contributed to the concept and design of the present study. JG was involved in data acquisition, analysis and interpretation. YM and YR drafted and critically revised the article for important intellectual content. All authors gave their approval for publication of the article. All authors agreed to be accountable for all aspects of the work in ensuring that questions related to the accuracy or integrity of the work are appropriately investigated and resolved.

\section{Ethics approval and consent to participate}

All procedures for animal care were approved by the Animal Management Committee of Qingdao University. All animal experiments were performed in compliance with the Guidelines for Proper Conduct of Animal Experiments, established by the Science Council.

\section{Patient consent for publication}

Not applicable.

\section{Competing interests}

The authors declare that they have no competing interests.

\section{References}

1. Wang D, Uhrin P, Mocan A, Waltenberger B, Breuss JM, Tewari D, Mihaly-Bison J, Huminiecki Ł, Starzyński RR, Tzvetkov NT, et al: Vascular smooth muscle cell proliferation as a therapeutic target. Part 1: Molecular targets and pathways. Biotechnol Adv 36: 1586-1607, 2018.

2. Alexander MR and Owens GK: Epigenetic control of smooth muscle cell differentiation and phenotypic switching in vascular development and disease. Annu Rev Physiol 74: 13-40, 2012.

3. Francis DJ, Parish CR, McGarry M, Santiago FS, Lowe HC, Brown KJ, Bingley JA, Hayward IP, Cowden WB, Campbell JH, et al: Blockade of vascular smooth muscle cell proliferation and intimal thickening after balloon injury by the sulfated oligosaccharide PI-88: Phosphomannopentaose sulfate directly binds FGF-2, blocks cellular signaling, and inhibits proliferation. Circ Res 92: e70-77, 2003.

4. Owens GK, Kumar MS and Wamhoff BR: Molecular regulation of vascular smooth muscle cell differentiation in development and disease. Physiol Rev 84: 767-801, 2004.

5. Gabbiani G, Schmid E, Winter S, Chaponnier C, de Ckhastonay C, Vandekerckhove J, Weber K and Franke WW: Vascular smooth muscle cells differ from other smooth muscle cells: Predominance of vimentin filaments and a specific alpha-type actin. Proc Natl Acad Sci USA 78: 298-302, 1981.

6. Hayashi K, Takahashi M, Nishida W, Yoshida K, Ohkawa Y, Kitabatake A, Aoki J, Arai H and Sobue K: Phenotypic modulation of vascular smooth muscle cells induced by unsaturated lysophosphatidic acids. Circ Res 89: 251-258, 2001.

7. Finney AC and Orr AW: Guidance molecules in vascular smooth muscle. Front Physiol 9: 1311, 2018.

8. Low EL, Baker AH and Bradshaw AC: TGF $\beta$, smooth muscle cells and coronary artery disease: A review. Cell Signal 53: 90-101, 2018.

9. Rikiyama T, Curtis J, Oikawa M, Zimonjic DB, Popescu N, Murphy BA, Wilson MA and Johnson AC: GCF2: Expression and molecular analysis of repression. Biochim Biophys Acta 1629: $15-25,2003$.

10. Ohtsuka H, Oikawa M, Ariake K, Rikiyama T, Motoi F, Katayose Y, Unno $M$ and Johnson AC: GC-binding factor 2 interacts with dishevelled and regulates Wnt signaling pathways in human carcinoma cell lines. Int J Cancer 129: 1599-1610, 2011.

11. Reed AL, Yamazaki H, Kaufman JD, Rubinstein Y, Murphy B and Johnson AC: Molecular cloning and characterization of a transcription regulator with homology to GC-binding factor. J Biol Chem 273: 21594-21602, 1998.

12. Li JP, Cao NX, Jiang RT, He SJ, Huang TM, Wu B, Chen DF, Ma P, Chen L, Zhou SF, et al: Knockdown of GCF2/LRRFIP1 by RNAi causes cell growth inhibition and increased apoptosis in human hepatoma HepG2 cells. Asian Pac J Cancer Prev 15: 2753-2758, 2014 
13. Chilakamarthi U, Koteshwar D, Jinka S, Vamsi Krishna N, Sridharan K, Nagesh N and Giribabu L: Novel amphiphillic G-quadruplex binding synthetic derivative of TMPyP4 and its effect on cancer cell proliferation and apoptosis induction. Biochemistry 57: 6514-6527, 2018.

14. Abbastabar M, Kheyrollah M, Azizian K, Bagherlou N, Tehrani SS, Maniati M and Karimian A: Multiple functions of p27 in cell cycle, apoptosis, epigenetic modification and transcriptional regulation for the control of cell growth: A double-edged sword protein. DNA Repair (Amst) 69: 63-72, 2018.

15. Fang H, Yang S, Luo Y, Zhang C, Rao Y, Liu R, Feng Y and Yu J: Notoginsenoside R1 inhibits vascular smooth muscle cell proliferation, migration and neointimal hyperplasia through PI3K/Akt signaling. Sci Rep 8: 7595, 2018.

16. Li XG and Wang YB: SRPK1 gene silencing promotes vascular smooth muscle cell proliferation and vascular remodeling via inhibition of the PI3K/Akt signaling pathway in a rat model of intracranial aneurysms. CNS Neurosci Ther 25: 233-244, 2018.

17. Very N, Vercoutter-Edouart AS, Lefebvre T, Hardiville S and El Yazidi-Belkoura I: Cross-Dysregulation of O-GlcNAcylation and PI3K/AKT/mTOR Axis in human chronic diseases. Front Endocrinol (Lausanne) 9: 602, 2018.

18. Mi XJ, Hou JG, Wang Z, Han Y, Ren S, Hu JN, Chen C and Li W: The protective effects of maltol on cisplatin-induced nephrotoxicity through the AMPK-mediated PI3K/Akt and p53 signaling pathways. Sci Rep 8: 15922, 2018.

19. Livak KJ and Schmittgen TD: Analysis of relative gene expression data using real-time quantitative PCR and the 2(-Delta Delta C(T)) method. Methods 25: 402-408, 2001

20. Khachigian LM, Santiago FS, Rafty LA, Chan OL, Delbridge GJ, Bobik A, Collins T and Johnson AC: GC factor 2 represses platelet-derived growth factor A-chain gene transcription and is itself induced by arterial injury. Circ Res 84: 1258-1267, 1999.

21. Suriano AR, Sanford AN, Kim N, Oh M, Kennedy S Henderson MJ, Dietzmann K and Sullivan KE: GCF2/LRRFIP1 represses tumor necrosis factor alpha expression. Mol Cell Biol 25: 9073-9081, 2005.

22. Song B, Liu X, Wang Q, Zhang R, Yang T, Han Z and Xu Y: Adenovirus-mediated shRNA interference against HSV-1 replication in vitro. J Neurovirol 22: 799-807, 2016.

23. Iyemere VP, Proudfoot D, Weissberg PL and Shanahan CM: Vascular smooth muscle cell phenotypic plasticity and the regulation of vascular calcification. J Intern Med 260: 192-210, 2006.
24. Zhang MJ, Zhou Y, Chen L, Wang YQ, Wang X, Pi Y, Gao CY, $\mathrm{Li}$ JC and Zhang LL: An overview of potential molecular mechanisms involved in VSMC phenotypic modulation. Histochem Cell Biol 145: 119-130, 2016.

25. Wang YQ, Chen C, Chen Z, Xu Y, Wang Y, Xiao BK, Chen SM and Tao ZZ: Indole-3-carbinol inhibits cell proliferation and induces apoptosis in Hep-2 laryngeal cancer cells. Oncol Rep 30: 227-233, 2013.

26. Bennetts PS and Pierce JD: Apoptosis: Understanding programmed cell death for the CRNA. AANA J 78: 237-245, 2010.

27. Shibley IA Jr, Gavigan MD and Pennington SN: Ethanol's effect on tissue polyamines and ornithine decarboxylase activity: A concise review. Alcohol Clin Exp Res 19: 209-215, 1995.

28. Lin SL, Chang DC, Ying SY, Leu D and Wu DT: MicroRNA miR-302 inhibits the tumorigenecity of human pluripotent stem cells by coordinate suppression of the CDK 2 and CDK4/6 cell cycle pathways. Cancer Res 70: 9473-9482, 2010.

29. Narasimha AM, Kaulich M, Shapiro GS, Choi YJ, Sicinski P and Dowdy SF: Cyclin D activates the Rb tumor suppressor by mono-phosphorylation. Elife: 3, 2014.

30. Karimian A, Ahmadi Y and Yousefi B: Multiple functions of p21 in cell cycle, apoptosis and transcriptional regulation after DNA damage. DNA Repair (Amst) 42: 63-71, 2016.

31. Ye G, Fu Q, Jiang L and Li Z: Vascular smooth muscle cells activate PI3K/Akt pathway to attenuate myocardial ischemia/reperfusion-induced apoptosis and autophagy by secreting bFGF. Biomed Pharmacother 107: 1779-1785, 2018.

32. Zhang Q, Chen L, Zhao Z, Wu Y, Zhong J, Wen G, Cao R, Zu X and Liu J: HMGA1 mediated high-glucose-induced vascular smooth muscle cell proliferation in diabetes mellitus: Association between PI3K/Akt signaling and HMGA1 expression. DNA Cell Biol 37: 389-397, 2018.

33. Zhang Q, Cao Y, Luo Q, Wang P, Shi P, Song C, E M, Ren J, Fu B and Sun $H$ : The transient receptor potential vanilloid-3 regulates hypoxia-mediated pulmonary artery smooth muscle cells proliferation via PI3K/AKT signaling pathway. Cell Prolif 51: e12436, 2018.

This work is licensed under a Creative Commons

Attribution-NonCommercial-NoDerivatives 4.0 International (CC BY-NC-ND 4.0) License. 